\title{
A DOAÇÃO DE SANGUE EM PROCEDIMENTOS CIRÚRGICOS: EXPERIÊNCIA EM UM SERVIÇO DE REFERÊNCIA EM TRANSPLANTE HEPÁTICO NO NORDESTE
}

\author{
Blood donation in surgical procedures: experience in a hepatic transplantation \\ reference service in the Northeast
}

\author{
Juliana dos Santos Gomes"; Maria José da Silva'; Eliane Souza Figueiredo"; Luiz Eduardo Rafael Moutinho²; \\ Olival Cirilo Lucena da Fonseca Neto3; Cláudio Moura Lacerda ${ }^{3}$
}

\begin{abstract}
RESUMO
Introdução: A utilização de produtos derivados do sangue desempenha um papel essencial na prática médica e a adequação dos estoques dos bancos de sangue é vital no impedimento de mortes evitáveis. A disponibilidade desses recursos depende absolutamente da doação de outro indivíduo e o recrutamento e a retenção de doadores de sangue pode ser um desafio de saúde pública. Objetivo: Este artigo objetiva reiterar a importância da intensificação de estratégias profissionais que possam, através de um público alvo específico, aumentar a oferta de hemocomponentes nos centros transfusionais. Métodos: Este estudo observacional transversal incluiu pacientes submetidos à abordagem cirúrgica, na unidade de transplante de fígado do Hospital Universitário Oswaldo Cruz (Recife/PE). Os dados sobre o perfil sociodemográfico, os procedimentos realizados e a utilização/reposição de hemoderivados foram analisados. Resultados: 35 pacientes foram entrevistados, dos quais $32(91,4 \%)$ fizeram uso de hemocomponentes. O transplante hepático foi absolutamente prevalente no grupo estudado (94,2\%). Dos pacientes que fizeram o uso de hemocomponentes, $77,1 \%$ não realizaram doação de reposição e todos (100\%) os pacientes que não a procederam alegaram a não solicitação profissional da instituição. Conclusão: Este estudo mostra a importância do planejamento institucional para a captação de doadores de sangue vinculados aos pacientes. De fato, a responsabilidade social e as campanhas educacionais são as estratégias chave no marketing relacionado à doação de sangue. Entretanto, a necessidade de articulação entre a demanda programada de doadores e o desenvolvimento de atividades coletivas e individuais deve ser exercitada em um cenário amplo do sistema de saúde, a fim de melhorar as práticas de doação de sangue.
\end{abstract}

Descritores: Doação de sangue, Estratégias, Procedimentos Cirúrgicos.

Instituições:

${ }^{1}$ Faculdade de Enfermagem da Universidade Maurício de Nassau, Recife/PE, Brasil.

2 Faculdade de Ciências Médicas da Universidade de Pernambuco, Recife/PE, Brasil.

${ }^{3}$ Hospital Universitário Oswaldo Cruz - HUOC, Recife/PE, Brasil.

\section{Correspondência:}

Olival Cirilo Lucena Fonseca Neto.

R. Jacobina, $n^{\circ} 45$ - Graças - CEP: 52011-180 Recife/PE

(81) 3184-1484

olivalneto@globo.com

Recebido em: 09/11/2019
Aceito em: 12/12/2019

\section{INTRODUÇÃO}

A utilização de produtos derivados do sangue desempenha um papel essencial na prática médica, e a adequação dos estoques dos bancos de sangue é vital no impedimento de mortes evitáveis, principalmente, em países subdesenvolvidos e em desenvolvimento. ${ }^{1}$ O avanço dos procedimentos médicos avançados, como transplantes e cirurgias de grande porte nessas regiões requerem, de fato, uma demanda crescente de hemocomponentes. A disponibilidade desse recurso depende absolutamente da doação de outro indivíduo (sendo este um ato altruísta, isento de qualquer tipo de remuneração, de acordo com as normas vigentes no Brasil) e o recrutamento e a manutenção de doadores de sangue podem ser um desafio de saúde pública. ${ }^{2}$ 
A literatura enfatiza o papel das campanhas específicas - focadas de acordo com a regionalidade e com o perfil do doador - no aumento do número de doações, na retenção do doador e na manutenção do suprimento estável de sangue.,3 Entretanto, alguns centros de coleta estimam que a contribuição dos primodoadores, aqueles que doam pela primeira vez, para fins de reposição, pode representar até $50 \%$ da oferta em algumas regiões do país. ${ }^{5}$ As políticas de captação de sangue no mundo, de acordo com o tipo de doação, comunitária ou de reposição, foram sendo modificadas ao longo da história e, desde a década de 1990, as principais autoridades sanitárias têm recomendado o incentivo ao doador de sangue comunitário e regular. ${ }^{6,7}$ A universalização dessa prática pode oferecer um suprimento mais constante, previsível, democrático e seguro do ponto de vista logístico e imunológico. ${ }^{5,8}$

Autores internacionais mostram que a análise da frequência de doação pode ser relacionada com a probabilidade de conversão do doador à repetição em longo prazo. ${ }^{3,9}$ Constata-se a forte associação entre gênero, idade, escolaridade, tipo de doador e solicitação da doação. Essas pesquisas são fundamentais para o ajuste das estratégias voltadas ao recrutamento e ao estímulo da regularidade das doações.

Tivemos o objetivo de analisar, portanto, a indicação da transfusão sanguínea nos pacientes submetidos à cirurgia em um centro de referência em transplante hepático localizado na Região Nordeste em relação à taxa de doação de reposição desses pacientes ao centro transfusional da região.

\section{MATERIAIS E MÉTODOS}

Trata-se de um estudo observacional transversal, realizado no ambulatório do serviço de cirurgia abdominal e transplante hepático do Hospital Universitário Oswaldo Cruz, localizado na Região Metropolitana do Recife (PE). Os pacientes submetidos a procedimento cirúrgico no serviço e que estavam regularmente em consulta ambulatorial durante o período do estudo foram incluídos.

As variáveis categóricas foram analisadas em termos de frequência absoluta e relativa. Os dados extraídos foram organizados através da Tabela 1 (dados sociodemográficos), Figura 1 (amostra dos procedimentos cirúrgicos) e Figura 2 (organograma do uso de hemocomponentes e doação de reposição).

Os dados foram coletados por meio de informações dos prontuários e através de um questionário previamente elaborado - aplicado presencialmente, mediante assinatura do Termo de Consentimento Livre e Esclarecido.
A pesquisa foi submetida ao comitê de ética em pesquisa da Universidade Maurício de Nassau (Recife/ PE), CAAE 49141915.4.0000.5193, e do Hospital Universitário Oswaldo Cruz (Recife/PE), CAAE 49141915.4.3001.5192.

\section{RESULTADOS}

Trinta e cinco pacientes foram entrevistados e os dados extraídos submetidos à análise. Houve predomínio da população do sexo masculino com faixa etária menor que 50 anos (Tabela 1). Foi constatado que $37,1 \%$ dos pacientes residem em outros estados e $94,2 \%$ foram submetidos ao transplante hepático (Figura 1).

Tabela 1: Dados Sociodemográficos

\begin{tabular}{|l|c|}
\hline \multicolumn{1}{|c|}{ Variáveis (N=35) } & $\mathbf{N ~ ( \% )}$ \\
\hline Sexo & $19(54.2)$ \\
\hline Homens & $16(45.7)$ \\
\hline Mulheres & \\
\hline Idade & $21(60)$ \\
\hline 0 a 50 anos & $14(40)$ \\
\hline 51 a 80 anos & \\
\hline Estado de origem & $22(62.8)$ \\
\hline Pernambuco & $13(37.1)$ \\
\hline Outros estados & \\
\hline
\end{tabular}

O organograma (Figura 2) mostra a demanda de uso dos hemocomponentes nas cirurgias: dos 35 pacientes entrevistados, $32(91,4 \%)$ realizaram uso de hemocomponentes enquanto três $(8,5 \%)$ não o fizeram. Dos $91 \%$ com necessidade de hemocomponentes, $77,1 \%$ não realizaram doação de reposição. Todos (100\%) os que não realizaram a reposição ao banco de sangue alegaram a não solicitação profissional da instituição, durante a entrevista realizada pela pesquisa.

Figura 1: Amostra dos Procedimentos Cirúrgicos

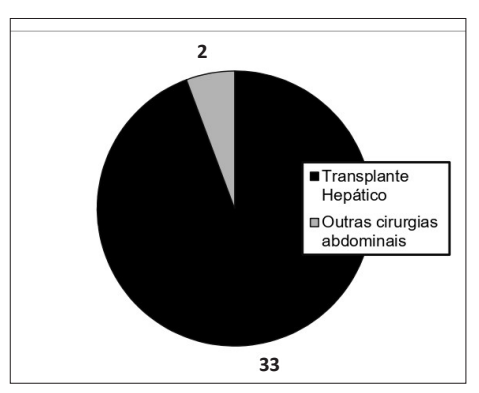


Juliana dos Santos Gomes; Maria José da Silva; Eliane Souza Figueiredo; Luiz Eduardo Rafael Moutinho; Olival Cirilo Lucena da Fonseca Neto; Cláudio Moura Lacerda

O organograma (Figura 2) mostra a demanda de uso dos hemocomponentes nas cirurgias: dos 35 pacientes entrevistados, $32(91,4 \%)$ realizaram uso de hemocomponentes enquanto três $(8,5 \%)$ não o fizeram. Dos $91 \%$ com necessidade de hemocomponentes,
$77,1 \%$ não realizaram doação de reposição. Todos $(100 \%)$ os que não realizaram a reposição ao banco de sangue alegaram a não solicitação profissional da instituição, durante a entrevista realizada pela pesquisa.

Figura 2: Organograma do Uso/Reposição de Hemocomponentes

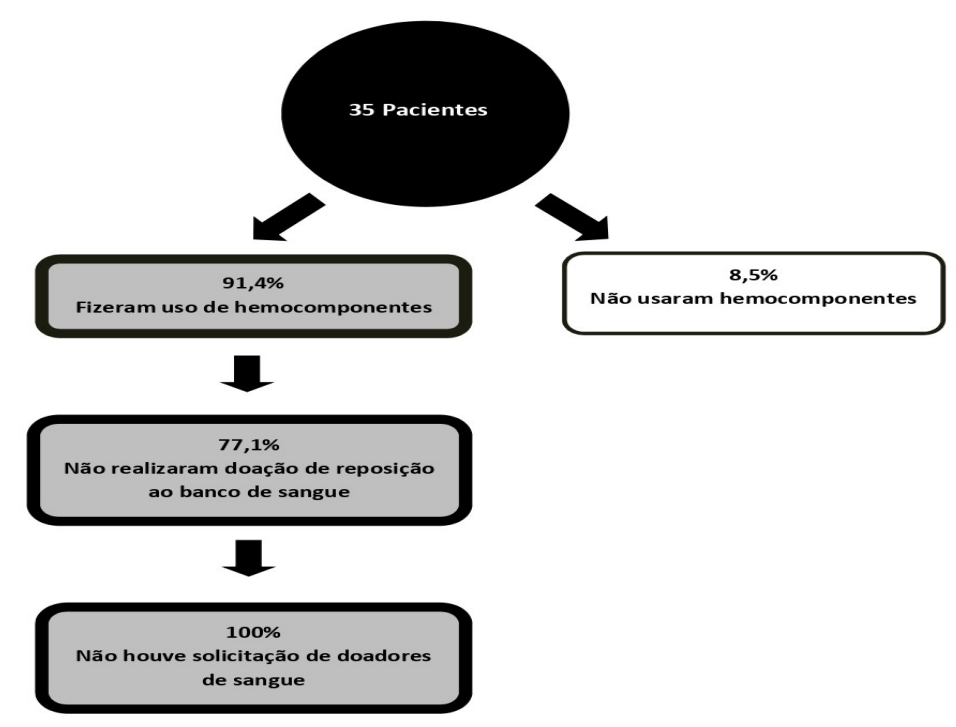

\section{DISCUSSÃO}

A participação voluntária na doação de sangue é fundamental para a manutenção regular dos estoques, buscando evitar que a demanda seja maior que a taxa de reposição de sangue. A doação de reposição compulsória (d̄eterminada pela instituição detentora da realização do procedimento cirúrgico) foi predominante, durante a década de 1980, e não foi eficaz, do ponto de vista comunitário aos estoques dos bancos de sangue. 5 Entretanto, obstáculos e exigências na captação e na qualidade de mais produtos sanguíneos convivem no cenário atual, havendo a doação de reposição uma incumbência ainda inevitável. ${ }^{10}$

Inicialmente, a institucionalização de estratégias orientadas para reposição pode ter um papel importante: A perspectiva da solidariedade no cenário da doação como um gesto inicial vinculado a outro indivíduo (que irá se submeter a um procedimento cirúrgico como exemplificado neste trabalho) pode contribuir com a intenção de, posteriormente, habituar os candidatos à solidariedade orgânica. Esse é um fenômeno espontâneo e desprovido de direcionamento específico, capaz de oferecer aos centros a adequação regular de hemocomponentes necessária. ${ }^{11}$ De fato, ao redor do mundo, estima-se que, cerca de $50 \%$ dos primodoadores não retornam a doar sangue e, então, ocorre aumento do número de publicações na literatura acerca das estratégias de captação e retenção de novos doadores: fenômeno importante para capilarização de tais práticas e solução de tal problema. 2,12,13

As principais estratégias de aumento da oferta de sangue são voltadas à qualidade do acolhimento e à educação social. O papel do profissional de saúde, na fidelização do doador através da comunicação e do desenvolvimento de um atendimento humanitário, é bastante compreendido no cenário do centro de hemotransfusão, ${ }^{14}$ assim como as campanhas educacionais voltadas à conscientização do altruísmo. ${ }^{9}$ Porém, sabe-se, também, que a captação de novos doadores é aumentada quando há solicitação direta ou incentivo de amigos e familiares. ${ }^{4,15}$ Esse tipo de estratégia pode ser transferida e institucionalizada nos serviços de cirurgia, a fim de promover a inicialização de diversos potenciais doadores de sangue.

A compreensão dos efeitos da comunicação, efetuada pelas instituições sobre a doação de sangue junto aos indivíduos, é indispensável no momento em que estas se propõem a formar uma cultura estruturada, em relação à necessidade da doação. ${ }^{16}$ 
Em outras palavras, o convencimento do doador de sangue à regularidade pode ser introduzido e institucionalizado. A menção à utilidade da doação como um ato altruísta e orgânico à vida e o entendimento dela como uma responsabilidade comunitária pode contribuir para regularidade da doação de sangue aliado, sobretudo, através de uma experiência pessoal. ${ }^{12}$ Observou-se, no hemocentro de Florianópolis, uma tendência de aumento das doações espontâneas em detrimento das doações de reposição, ao longo dos anos de 1989 a 2008.

O reconhecimento da importância das ações institucionais e da orientação do doador é fundamental quando se pensa em estratégias para aumentar a retenção de primodoadores. Por meio dessa direção, as instituições podem tentar identificar estratégias e motivações para incentivar a doação de sangue diante de diversos cenários da rede de saúde. Devese destacar, ainda, que um elemento comum às organizações é o aspecto, de maneira essencial, do foco no usuário e da comunicação interpessoal no almejo desse objetivo.

\section{CONCLUSÃO}

As instituições têm importância social chave na captação de doadores, principalmente, por atender pacientes que não sobreviveriam sem a devida reposição. Os autores deste trabalho sugerem a necessidade de esforços coordenados para aumentar a doação de sangue, tanto na captação de novos doadores, quanto na fidelização de doadores regulares.

\section{ABSTRACT}

Introduction: Using blood products plays an essential role in medical practice and adequacy of blood stocks is vital in preventable deaths. The availability of this resource absolutely depends on donation from another individual, and recruitment and retention of blood donors can be a public health challenge. Purpose: This article aims to reiterate the importance of intensifying professional strategies that can, through a specific targeted audience to increase supply of blood components in transfusion centers. Methods: This cross-sectional observational study included patients who underwent surgery in liver transplant unit at Hospital Universitário Oswaldo Cruz (Recife/PE). Data on sociodemographic profile, procedures performed and use/replacement of blood products were analyzed. Results: 35 patients were interviewed, of which $32(91.4 \%)$ used blood components. Liver transplantation was absolutely prevalent in the studied group (94.2\%). $77.1 \%$ of patients who used blood components did not make replacement donation and all $(100 \%)$ of then claimed it was not professionally requested by the institution. Conclusion: This study shows the importance of institutional planning for attracting blood donors linked to patients. In fact, social responsibility and educational campaigns are the key marketing strategies related to blood donation. However, coordination between programmed donor demand and development of collective and individual activities must be exercised in a broad context of the health system in order to improve blood donation practices.

Keywords: Blood donation, Strategies, Surgical Procedures 
Juliana dos Santos Gomes; Maria José da Silva; Eliane Souza Figueiredo; Luiz Eduardo Rafael Moutinho; Olival Cirilo Lucena da Fonseca Neto;

\section{REFERÊNCIAS}

1. Roberts N, James S, Delaney M, Fitzmaurice C. The global need and availability of blood products: a modelling study. Lancet Haematol. 2019;6(12):e606-15.

2. Rodrigues RSM, Reibnitz KS. Estratégias de captação de doadores de sangue: uma revisão integrativa da literatura. Texto Amp Contexto - Enferm. 2011;20(2):384-91.

3. Gonçalez TT, Di Lorenzo Oliveira C, Carneiro-Proietti ABF, Moreno EC, Miranda C, Larsen N, et al. Motivation and Social Capital among prospective blood donors in three large blood centers in Brazil. Transfusion (Paris). 2013;53(6):1291-301.

4. Ludwig ST, Rodrigues AC de M. Doação de sangue: uma visão de marketing. Cad Saúde Pública. 2005;21(3):9329.

5. Carneiro-Proietti A, Sabino E, Sampaio D, Proietti F, Gonçalez T, Oliveira C, et al. Demographic profile of blood donors in Brazil: Results from the International REDS II Study, 2007-2008. Transfusion (Paris). 2010;50(4):91825.

6. Pimentel MA. A questão do sangue: rumos das políticas públicas de hemoterapia no Brasil e no exterior [Internet]. Universidade do Estado do Rio de Janeiro. Instituto de Medicina Social; 2006 [citado 21 de julho de 2019]. Disponível em: http://bases.bireme.br/cgi-bin/wxislind.exe/ iah/online/?IsisScript=iah/iah. $x i s \& s r c=g o o g l e \& b a s e=$ LILA CS\&lang=p\&nextAction=Ink\&exprSearch=433583\&index Search $=$ ID

7. World Health Organization. Aide-memoire on Blood Safety for National Blood Programmes [Internet]. 1999. Disponível em: https://www.who.int/bloodsafety/ publications/en/
8. Barreto CC, Sabino EC, Gonçalez TT, Laycock ME, Pappalardo BL, Salles NA, et al. Prevalence, incidence, and residual risk of human immunodeficiency virus among community and replacement first-time blood donors in São Paulo, Brazil. Transfusion (Paris). 2005;45(11):1709-14.

9. Glynn SA, Kleinman SH, Schreiber GB, Zuck T, Combs SM, Bethel J, et al. Motivations to donate blood: demographic comparisons. Transfusion (Paris). 2002;42(2):216-25.

10. Bates I, Manyasi G, Medina Lara A. Reducing replacement donors in Sub-Saharan Africa: challenges and affordability. Transfus Med Oxf Engl. 2007;17(6):434-42.

11. Pereima RSMR, Reibnitz KS, Martini JG, Nitschke RG. Doação de sangue: solidariedade mecânica versus solidariedade orgânica. Rev Bras Enferm. 2010;63(2):322-7.

12. Bagot KL, Murray AL, Masser BM. How Can We Improve Retention of the First-Time Donor? A Systematic Review of the Current Evidence. Transfus Med Rev. 2016;30(2):81-91.

13. Chell K, Davison TE, Masser B, Jensen K. A systematic review of incentives in blood donation. Transfusion (Paris). 2018;58(1):242-54.

14. Giacomini L, Lunardi Filho WD. Estratégias para fidelização de doadores de sangue voluntários e habituais. Acta Paul Enferm. 2010;23(1):65-72.

15. Davey RJ. Recruiting blood donors: challenges and opportunities. Transfusion (Paris). 2004;44(4):597-600.

16. Ludwig ST. COMUNICAÇÃO DA DOAÇÃO DE SANGUE: UMA ANÁLISE SOB A ÓTICA DAS TEORIAS COGNITIVAS. Rev Estud Comun [Internet]. 2008;9(20). Disponível em: https://periodicos.pucpr.br/index.php/ estudosdecomunicacao/article/view/16598 